\title{
anu \\ Ultra-Low Power Wireless Sensor Networks Based on Time Slotted Channel Hopping with Probabilistic Blacklisting
}

\author{
Gianluca Cena (D), Stefano Scanzio *(D) and Adriano Valenzano (D) \\ National Research Council of Italy (CNR-IEIIT), Corso Duca degli Abruzzi 24, I-10129 Torino, Italy; \\ gianluca.cena@ieiit.cnr.it (G.C.); adriano.valenzano@ieiit.cnr.it (A.V.) \\ * Correspondence: stefano.scanzio@ieiit.cnr.it
}

check for updates

Citation: Cena, G.; Scanzio, S.; Valenzano, A. Ultra-Low Power Wireless Sensor Networks Based on Time Slotted Channel Hopping with Probabilistic Blacklisting. Electronics 2022, 11, 304. https://doi.org/ 10.3390 /electronics 11030304

Academic Editor: Akash Kumar

Received: 29 December 2021

Accepted: 17 January 2022

Published: 19 January 2022

Publisher's Note: MDPI stays neutral with regard to jurisdictional claims in published maps and institutional affiliations.

Copyright: (C) 2022 by the authors. Licensee MDPI, Basel, Switzerland. This article is an open access article distributed under the terms and conditions of the Creative Commons Attribution (CC BY) license (https:// creativecommons.org/licenses/by/ $4.0 /)$.

\begin{abstract}
Devices in wireless sensor networks are typically powered by batteries, which must last as long as possible to reduce both the total cost of ownership and potentially pollutant wastes when disposed of. By lowering the duty cycle to the bare minimum, time slotted channel hopping manages to achieve very low power consumption, which makes it a very interesting option for saving energy, e.g., at the perception layer of the Internet of Things. In this paper, a mechanism based on probabilistic blacklisting is proposed for such networks, which permits to lower power consumption further. In particular, channels suffering from non-negligible disturbance may be skipped based on the perceived quality of communication so as to increase reliability and decrease the likelihood that retransmissions have to be performed. The only downside of this approach is that the transmission latency may grow, but this is mostly irrelevant for systems where the sampling rates are low enough.
\end{abstract}

Keywords: probabilistic blacklisting; TSCH; adaptive channel capacity shaping; ACCS; WSN; ultra-low power

\section{Introduction}

Wireless sensor networks (WSNs) often include devices that are powered on batteries, especially when they are deployed in areas without a proper infrastructure. A primary requirement in these cases is to lower as much as possible the power consumption of such devices. Doing so brings two main benefits. First, the costs related to battery replacement, which also include the need for specialized personnel to physically access devices, decrease; it is worth noting that, sometimes, sensors can be located in places that are not very easy to reach. Second, the amount of exhausted batteries to be disposed of at the end of their life is proportionally reduced. Very simply, even a seemingly modest reduction in the power consumption by, say, $10 \%$, means that waste, which is likely to cause serious environmental pollution if not disposed of properly, is lowered to the same extent. It must be pointed out that also the solutions based on energy harvesting, including self-charging batteries [1], can definitely benefit from approaches aimed at saving energy.

The past decades have witnessed dramatic advances in low-power electronics, which made it possible to design and build smart objects and to enable the Internet of Things (IoT) [2]. Such objects are found at the border between the perception and network/transport layers in the IoT reference model, and their operations basically combine sensing and data transmission, usually toward a suitable middleware for subsequent processing in the Edge/Cloud. While a further reduction in power consumption is expected for them in the next years, some contributions can be hardly shrunk by acting only on the local processing platform (hardware, software, and operating system). This is the case of the energy required to transmit and receive signals over the air, exploiting, for instance, radio frequencies. In fact, decreasing the transmission power unavoidably makes the signals weaker against electromagnetic disturbance, consequently leading to a reduction in the packet delivery ratio, which may no longer fit the application requirements, undermining system reliability. 
Modulation techniques adopted at the physical layer of the protocol stack in the recent solutions are typically very energy efficient, and are not likely to be improved further in the near future $[3,4]$. This means that other approaches are needed to save energy without impairing communication reliability, which operate at the medium access control (MAC) layer. One of the most popular techniques conceived to this purpose is time slotted channel hopping (TSCH) [5-7]. In this case, all motes are time synchronized and transmissions take place at predefined instants, which are known to all the participants of any communication. This implies that receivers are not requested to be always on. Instead, they can go to sleep until the time a message relevant to them is (potentially) expected. In simple words, TSCH manages to dramatically cut down power consumption on the receiving side by reducing the duty cycle. Other mechanisms, such as the deterministic and synchronous multi-channel extension (DSME), are basically aimed at the same purpose, although they do not require time synchronization [8].

Several proposals have been defined in the recent past that permit further saving. Some exploit a priori knowledge about future data exchanges. This is the case, for example, for cyclic data transfers, whose period can be inferred automatically and exploited to make receivers sleep for longer times. In [9], a simple mechanism is proposed for the MAC layer, termed listening suspension, which permits the transmitting side of a TSCH link to put the receiver to sleep for a given amount of time. The proactive reduction of idle listening (PRIL) $[10,11]$ proposal generalizes such an approach, by exploiting information collected from different protocol layers in such a way as to tackle also multi-hop paths. Instead, other techniques operate explicitly at the upper protocol layers. For instance, in [12] two heuristics based on gravitational approaches were proposed for clustering and routing algorithms in WSNs, which rely on a fuzzy logic. In [13], the effects of inter-node distance and packet length on the network lifetime were analyzed, and it was found that, to cover a certain area, it is advisable to increase the packet length and to reduce the number of nodes.

One of the main TSCH strengths is channel hopping: in fact, while time slotting permits to prevent intra-network collisions and to increase energy efficiency, channel hopping makes transmission more robust against narrowband noise and external interference, which usually affects a limited number of frequencies among those that TSCH is allowed to use. For example, in the $2.4 \mathrm{GHz}$ industrial, scientific, and medical (ISM) band, 16 channels, numbered from 11 to 26 , are actually available. The transmission frequency typically hops among this set of channels according to a predefined sequence known by all motes. The automatic repeat request (ARQ) mechanism, which keeps retransmitting a frame until either the related acknowledgment frame (ACK) is received from the destination or the maximum number of transmission attempts, as given by the retry limit $\left(R_{\mathrm{L}}\right)$, is exceeded, is layered atop TSCH. If the number of available channels and the slotframe size are selected in a suitable way, every subsequent retry is performed on a different channel, thus providing a very effective frequency diversity [14]. Generally speaking, the likelihood for transmission attempts in TSCH to take place on any of the different channels is, in the long term, approximately the same.

Including in TSCH, some mechanism that makes the usage of channels depend on their quality would lower, on average, the number of performed retries since good channels are used more often than bad ones. As a consequence, doing so could lower power consumption, improving at the same time reliability (the allowed number of attempts remains the same, but the likelihood for them to succeed increases). Generally speaking, blacklisting is a very popular mechanism that relies on the above idea. By temporarily excluding channels that suffer from high disturbance, the overall network performance typically improves. Most blacklisting techniques conceived for TSCH are aimed at improving the quality of communication, on either the network or a single link, which means achieving a higher packet delivery ratio (PDR) and lower latency. For example, in [15] the LABeL technique was proposed for the 6TiSCH protocol stack [6], which implements IPv6 over the TSCH mode of IEEE 802.15.4e. This approach exploits link-based adaptive blacklisting in 
order to improve reliability and responsiveness for industrial applications, where latency does definitely count.

In this paper, we consider quite different scenarios, where minimizing latency is not a primary requirement. Therefore, what we will actually do is trading responsiveness for power consumption. In particular, we do the following:

- Our main goal is to reduce the amount of energy spent for exchanging frames over a TSCH link, even if this causes the communication latency to grow.

- An equally important goal is not to worsen reliability, which means that the PDR on each link (and, consequently, over the network) must be, on average, as high as TSCH (or better).

- In addition, we ask that situations where communication is prevented (or impaired) because the state seen by the involved motes is no longer coherent must be avoided: since the mechanism we propose operates on a per-link basis, there is no need to propagate any information about blacklisting to other motes, which prevents this kind of issue.

- Finally, we pursue a very simple mechanism, as motes are very often provided with a limited amount of processing power and memory.

The paper is structured as follows: in Section 2 a brief introduction is provided about TSCH and blacklisting, whereas our adaptive approach is described in Section 3. Section 4 is aimed at evaluating the ability of moving average techniques to estimate the failure probability of channels, while a thorough performance evaluation of the proposed approach is provided in Section 5. Finally, in Section 6, some conclusions are drawn.

\section{Improving TSCH through Blacklisting}

Below, we focus on WSNs, which are used to reliable acquire information from sensors connected over the air [16]. Although we specifically consider TSCH, the proposed approach can be applied with some modification to other communication protocols as well. We assume that sampling periods are large enough, in the order of several minutes up to a few hours. What we demand is to reduce power consumption implied by frame exchanges to the bare minimum, leaving communication reliability (that is, the likelihood that a frame is eventually delivered to destination successfully) as high as conventional TSCH. We do not consider explicitly the option to bring modifications to the physical layer, including optimizations concerning the number and position of motes. In fact, such enhancements are usually independent from those brought to the MAC layer, and both can be adopted jointly to achieve the best results.

\subsection{TSCH Basics}

Basically, the TSCH medium access relies on time slotting. Frame exchanges are driven by a slotframe that repeats cyclically to which all motes must synchronize. The slotframe consists of a fixed number of slots, and every slot is wide enough to contain a maximal data frame, plus, if required, the related ACK. For instance, for OpenMote B devices running OpenWSN, the default slot duration is $T_{\text {slot }}=20 \mathrm{~ms}$. Scheduling of transmission opportunities in TSCH is achieved through a matrix made up of $N_{C}$ rows and $N_{S}$ columns, where $N_{\mathrm{C}}$ is the number of physical channels over which frequency hops and $N_{\mathrm{S}}$ is the number of slots in the slotframe. As said above, when operating in the $2.4 \mathrm{GHz}$ band, $N_{\mathrm{C}}$ is customarily set to 16 . The default setup for the above platform foresees $N_{S}=101$, which implies that the slotframe duration is $2.02 \mathrm{~s}$, but this parameter can be easily changed.

Every dedicated link between a transmitter and a receiver mote is allocated one (or more) specific cells, each one identified by a pair $\left\langle S_{\text {off }}, C_{\text {off }}\right\rangle$ that represents its position in the TSCH matrix (slot and channel offsets, respectively). The channel used for transmission in the cell $\left\langle S_{\text {off }}, C_{\text {off }}\right\rangle$ is not fixed. Instead, it is calculated at runtime starting from index $\left(A S N+C_{\text {off }}\right) \% N_{C}$, where "\%" denotes the modulo operator and $A S N$ is a 5-byte integer that represents the absolute slot number. $A S N$ is initialized to 0 at network startup and is increased by one on every slot. By selecting $N_{\mathrm{C}}$ and $N_{\mathrm{S}}$ as prime numbers, consecutive 
transmissions on any link (and, in particular, retries for the same frame) take place on different channels [17], effectively enabling mixed time-frequency diversity.

A suitably designed bijective function, modeled as an array $H$, maps every above index onto a distinct physical channel $c$. In practice, $c=H\left[\left(A S N+C_{\text {off }}\right) \%|H|\right]$, where the size $|H|$ of the hopping sequence is customarily set equal to $N_{C}$. Doing so permits to increase communication robustness in those situations, where a number of frequencies may be affected at the same time by the same phenomenon [18]. This is the case of the traffic generated by nearby Wi-Fi devices: in fact, every single $20 \mathrm{MHz}$ Wi-Fi channel affects four adjacent TSCH channels. Higher pseudo-randomness in channel hopping better counteracts this kind of interference, irrespective of the actual (coprime) values chosen for $N_{C}$ and $N_{S}$. The notation used throughout the text is summarized in Table 1.

Table 1. Summary about notation.

\begin{tabular}{lc}
\hline Quantity & Symbol \\
\hline Slotframe size (in slots) & $N_{\mathrm{S}}$ \\
Time slot duration & $T_{\text {slot }}$ \\
Slotframe duration & $T_{\mathrm{sf}}$ \\
Absolute slot number & $A S N$ \\
Slot offset in the TSCH matrix for a given cell & $S_{\text {off }}$ \\
\hline Number of channels & $N_{\mathrm{C}}$ \\
Hopping sequence & $H[\cdot]$ \\
Channel offset in the TSCH matrix for a given cell & $C_{\text {off }}$ \\
\hline Number of quantization levels & $N_{Q}$ \\
Function for making $q^{\prime}$ pseudo-random & $Q[\cdot]$ \\
\hline Sampling windows width for SMA low-pass filter & $M$ \\
Aging factor (weight) for EMA low-pass filter & $\alpha$ \\
\hline Outcome for attempt $i$ on channel $c$ (fail. $=1$, succ. $=0)$ & $\phi_{c}^{(i)}$ \\
Real failure probability for channel $c$ & $\epsilon_{c}$ \\
Estimated failure probability for channel $c$ & $\hat{\epsilon}_{c}$ \\
Quantized disturbance for channel $c$ & $q_{c}$ \\
Normalized quantized disturbance for channel $c$ & $q_{c}^{\prime}$ \\
\hline Retry limit & $R_{\mathrm{L}}$ \\
Number of transmission attempts for frame $j$ & $a_{j}$ \\
Number of skipped cells for frame $j$ & $s_{j}$ \\
Transmission latency for frame $j$ & $d_{j}$ \\
Upper bound on $d_{j}$ (in slotframes) & $\hat{\delta}_{j}$ \\
\hline
\end{tabular}

\subsection{Channel Blacklisting}

The basic idea behind channel blacklisting techniques in TSCH is to change the hopping sequence $H$ to prevent transmissions from occurring on poor quality channels. Unfortunately, this seemingly simple idea leads to a number of non-negligible issues when applied in practice. Performing channel evaluation once and for all at network startup is ineffective, as spectrum conditions are likely to vary over time (movement of motes and absorbing/reflecting obstacles, variations in the interfering traffic and electromagnetic noise, etc.). This means that spectrum conditions have to be repeatedly evaluated at runtime, exploiting adaptive approaches. This leads to the question about how evaluation has to be performed. Some approaches rely on the received signal strength indication (RSSI), which is readily available on the receiver side [19]. As an alternative, the failure probability on every channel can be estimated on the transmitting side as the ratio between the number of transmission attempts for which an ACK frame is received and the overall number of attempts performed by the mote [15], only considering cells mapped by TSCH on that particular channel. 
A second aspect to be taken into account is how measurements about the spectrum conditions, carried out by a plurality of motes, are used to change the hopping sequence. Although many clever solutions have been proposed for this optimization problem [20-22], several constraints must be faced:

1. A small amount of energy must be spent by motes for computing the new hopping list, which means that both the complexity of the blacklisting algorithm and the number of additional messages needed to carry out its duties must be kept as low as possible.

2. The time taken to perform spectrum characterization, to evaluate the new optimal hopping sequence and to distribute it to the motes should be as low as possible, so that the ever-changing conditions of the wireless medium can be tracked effectively, yielding tangible improvements.

3. All other conditions met, a simple and robust mechanism, which is intrinsically stable and unlikely leads to oscillating or inconsistent behavior, is usually sought.

From an architectural point of view, several options are available. Centralized approaches, where a single node (typically, the gateway) collects measurements about the spectrum conditions from the motes and computes a new optimal global hopping sequence, are robust but slow, and may be ineffective. Distributed approaches leave the optimization burden to the motes, which have to communicate with neighbors to prevent clashes in channel reallocation (which would lead to collisions). Doing so permits to perform local optimizations, possibly exploiting channel re-use when links are located far away one from another. However, ensuring high robustness for dynamically changing environments could be quite troublesome in these cases, and may require a substantial number of control frames to be exchanged with nearby motes, which increase power consumption.

Concerning the specific way the hopping sequence $H$ is dynamically updated, different solutions are possible. In the most general case, the size of the hopping list can be changed at runtime. Typically, it is shortened when a bad channel is discovered, which is removed from the list. Blacklisted channels are re-enabled when they are discovered to behave satisfactorily again. This approach has a non-negligible drawback: if, for any reason, coherence about $H$ (as seen by motes) is lost, communication is prevented almost completely, because transmitters and receivers no longer agree on the channel to be used for any given $A S N$ value. This is due to the fact that periodicity in channel pseudo-random selection depends on the hopping list size (according to the modulo operator), which is no longer fixed (motes only know that $|H| \leq N_{\mathrm{C}}$ ).

A more robust solution is to replace any bad channel with a good one (which is hence used more often), which leaves the length of the hopping sequence unchanged. In this case, if coherence is lost, communication is affected only on the replaced channels. In particular, collisions may take place whenever more than one mote in the same area is enabled to transmit in the same slot and on the same channel. If at most one mote is scheduled for transmission in the same slot (i.e., when no more than one link is defined in every column of the TSCH matrix), such an event cannot occur. The price to pay, in this case, is the low number of available links (limited by $N_{S}$ ), which decreases the maximum number of motes that can be connected to the network. On the other hand, if multiple motes are scheduled for transmission in the same slot on different channels (exploiting $C_{\text {off }}$ parameters), care has to be taken so that no two of them, as part of the update process for $H$ involved by blacklisting, can select the same replacement channels, which would lead to collisions.

The simplest and most intrinsically robust blacklisting technique is to just skip transmission in those cells for which the quality of the related physical channel is deemed not good enough. For example, a threshold can be defined to this purpose. Since we are pursuing simplicity and robustness, this kind of approach will be specifically investigated.

\section{Adaptively Shaping the Capacity of Channels}

Measuring channel quality and disabling transmission completely when it falls below a given threshold is probably not the best solution, since intervention is not gradual but abrupt. Moreover, when a channel is disabled, its quality is not assessed any longer, and it 
cannot be easily reintegrated. For this reason, a pseudo-probabilistic approach is defined. The adaptive channel capacity shaping(ACCS) solution we are proposing operates on a per-link basis, and consists in selectively tuning the amount of transmission opportunities provided on every physical channel based on the related quality of communication. To put it simple, if the quality of a channel is high, every cell mapped on that channel will be available for transmission (and will be used, provided that the related link has a pending frame to be sent). Conversely, the lower the quality of the channel, the lower the chance that a cell mapped on it can be actually used.

As depicted in Figure 1, the ACCS operation can be described through a number of blocks, which (in line of principle) are implemented only on the transmitting side of the link.

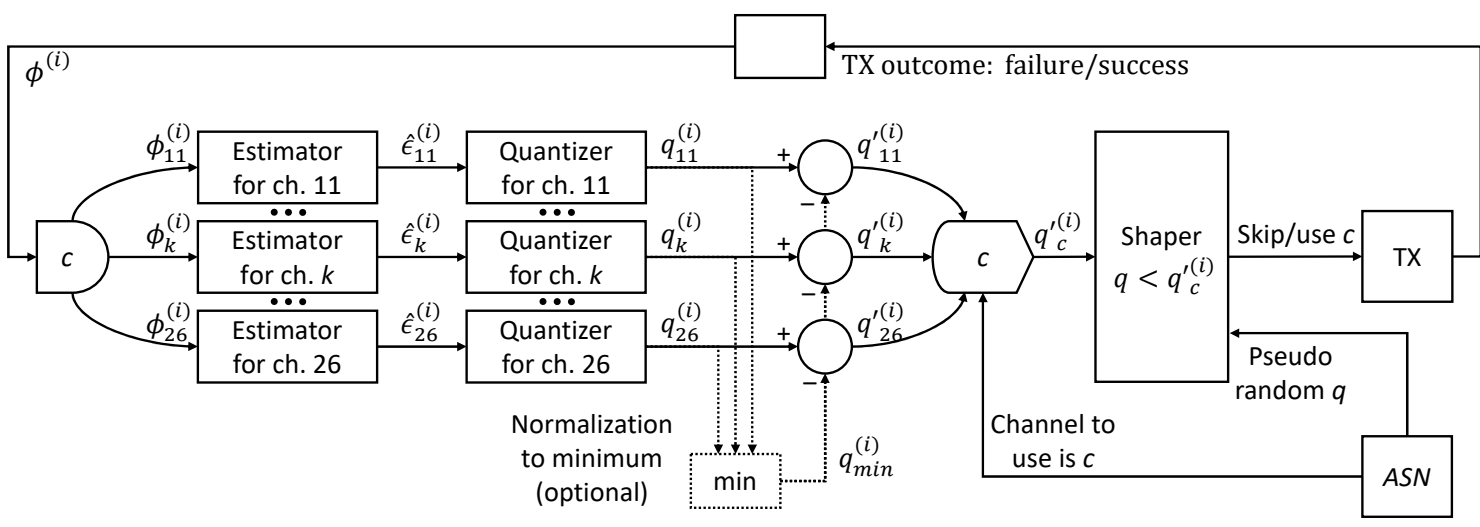

Figure 1. Block diagram for ACCS (including optional normalization to the minimum): the MAC of $\mathrm{TSCH}$ is instructed whether to use or to skip every cell allocated to the link.

\subsection{Channel Quality Estimation}

The estimator block is aimed at measuring, for any given link, how good every physical channel is. In the simple implementation we are considering here, the sender checks the outcome of every confirmed transmission attempt performed on link $\ell$ to estimate at runtime the failure probability $\epsilon_{\ell, c}$ on every channel $c$. To simplify descriptions, a single link is considered in the following, so that the streamlined notation $\epsilon_{\mathcal{C}}$ can be used. It coincides with the probability that, for a single transmission attempt (either the initial attempt or one of the retries) on channel $c$, the related ACK frame is not received. When several cells in the slotframe are allocated for transmission toward the same destination (over-provisioning), the related statistics can be gathered together. This is not possible for frames targeted to different destinations, even if they are scheduled in the same cell (e.g., for downlinks), as the physical propagation path for them is likely not the same. For the sake of simplicity, the term link will be used in the following to denote the communication between a specific source mote and a specific destination mote. Since the ACCS mechanism operates on a per-link basis, only the destination is needed to identify the link in each sender node.

Let $i$ denote the most recent transmission attempt performed on channel $c$, and let $\phi_{c}^{(i)}$ be the related outcome (either 0 for success or 1 for failure). A simple moving average (SMA) can be used to estimate the failure probability at time $t_{i}$

$$
\hat{\epsilon}_{c}^{(i)}=\frac{1}{M} \sum_{j=0}^{M-1} \phi_{c}^{(i-j)},
$$

where $M$ is the number of past samples to be stored by the estimator block per link and per channel, e.g., using a circular buffer. Although using SMA to obtain $\epsilon_{c}$ from $\phi_{c}$ samples resembles a finite impulse response (FIR) low-pass filter, they are not exactly the same. In fact, frame transmissions in WSNs are typically periodic, but retries are not. However, if the 
spectrum dynamics are slow enough, the ability of SMA to track its variations adequately is not impaired significantly.

As an alternative, an exponential moving average(EMA) can be exploited. In this case,

$$
\begin{aligned}
& \hat{\epsilon}_{c}^{(i)}=\alpha \phi_{c}^{(i)}+(1-\alpha) \hat{\epsilon}_{c}^{(i-1)}, \\
& \hat{\epsilon}_{c}^{(0)}=0,
\end{aligned}
$$

where $\hat{\epsilon}_{c}^{(i-1)}$ denotes the previous $\epsilon_{c}$ estimate. This approach resembles the one used to evaluate the retransmission timer in TCP [23], and is conceptually based on a first-order infinite impulse response (IIR) low-pass filter (the same considerations made above apply).

\subsection{Disturbance Discretization}

The output $\hat{\epsilon}_{c}$ of the estimator block, which in embedded devices can be expressed as a fixed-point value in the range $[0,1]$, is first fed into a quantizer block with $N_{\mathrm{Q}}$ quantization levels to convert it to an integer $q_{c}$. In our case,

$$
q_{c}=\min \left(\left\lfloor\hat{\epsilon}_{c} \cdot N_{\mathrm{Q}}\right\rfloor, N_{\mathrm{Q}}-1\right),
$$

so that $q_{c} \in\left[0, N_{\mathrm{Q}}-1\right]$. In particular, $q_{c}=0$ denotes a good quality channel, whereas $q_{c}=N_{\mathrm{Q}}-1$ denotes a very poor quality channel (including the case when all transmissions performed on $c$ fail and $\hat{\epsilon}_{c}=1$, e.g., in the presence of a narrowband jamming signal).

Non-linear laws for converting $\hat{\epsilon}_{c}$ to $q_{c}$ are also possible, but we did not consider them here. We set $N_{\mathrm{Q}}=9$, so that the link quality (in terms of the disturbance level) can be encoded on four bits only. Finer discretization on a larger number of levels just achieved marginal improvements.

\subsection{Channel Capacity Shaping}

Disturbance levels for all channels, defined by the set $\left\{q_{k}\right\}_{k \in[11,26]}$, are finally fed as input to a shaper block. Let $c$ be the channel used by TSCH for transmitting in the current slot (in every slot, at most one link is enabled for transmission on any given mote). The purpose of the shaper is to selectively skip part of the cells by choking every channel of the considered link to a fraction $1-\left(q_{c} / N_{\mathrm{Q}}\right)$ of the whole capacity based on its quality. To this extent, an integer quantity $q$ is defined in the shaper, evaluated as $q=A S N \% N_{\mathrm{Q}}$. It is important that $N_{\mathrm{Q}}, N_{\mathrm{C}}$, and $N_{\mathrm{S}}$ are prime numbers. This means that, by considering any $N_{\mathrm{Q}}$ consecutive cells of the same link whose transmission is set to happen on channel $c$, all of them are characterized by different $q$ values. Moreover, the related sequence of $q$ values repeats itself every $N_{\mathrm{Q}}$ such cells, i.e., every $N_{\mathrm{Q}} \cdot N_{\mathrm{C}} \cdot N_{\mathrm{S}}$ slots. In a real implementation, a bijective function $Q$ should be additionally applied, that is, $q^{\prime}=Q\left[A S N \% N_{\mathrm{Q}}\right]$, so that the $q^{\prime}$ values seemingly hop in a truly random way across all the values in the range $\left[0, N_{\mathrm{Q}}-1\right]$.

The $q_{c}$ value is interpreted by the shaper as the number of cells of the link, by considering those mapped on channel $c$, to be skipped every $N_{\mathrm{Q}}$ cells. To determine if the current cell associated to channel $c$ has to be skipped, the very simple test $q^{\prime}<q_{c}$ is performed. The net effect is a pseudo-random culling of the transmission opportunities, driven by the disturbance level. As an example, Figure 2 shows, for every possible value of $q_{c}$ (each one taking one column in the rightmost part of the figure), the sequence of cells skipped for channel $c$, under the assumption that (a) $N_{S}=101$ and $N_{C}=16$ (which means that the link transmits on the same channel every 1616 slots); (b) $N_{\mathrm{Q}}=9$; (c) the cell is located at $S_{\text {off }}=1$; and, (d) $C_{\text {off }}$ is such that channel $c$ is selected for transmission (through $H$ ) for $A S N=1$ and after every multiple of 1616 slots, that is, $H\left[\left(1+C_{\text {off }}\right) \% 16\right]=c$. A very simple $Q$ mapping is defined to obtain $q^{\prime}$. As can be easily seen, when $q_{c}=0$ (on the left) no cells are skipped, whereas when $q_{c}=N_{\mathrm{Q}}-1=8$ (on the right), only one cell out of $N_{\mathrm{Q}}$ consecutive ones is available for transmission on the link. In between, a linear law (better, a staircase) relates the fraction of skipped cells and the failure rate for any given channel, hence achieving smooth operations. Other heuristics are clearly possible. 


\begin{tabular}{|c|c|c|c|c|c|c|c|c|c|c|c|c|}
\hline$A S N$ & $q=A S N \% 9$ & $q^{\prime}=Q(q)$ & $q_{c}$ & 0 & 1 & 2 & 3 & 4 & 5 & 6 & 7 & 8 \\
\hline 1 & 1 & 1 & & & & Skip & Skip & Skip & Skip & Skip & Skip & Skip \\
\hline 1,617 & 6 & 6 & & & & & & & & & Skip & Skip \\
\hline 3,233 & 2 & 3 & & & & & & Skip & Skip & Skip & Skip & Skip \\
\hline 4,849 & 7 & 8 & & & & & & & & & & \\
\hline 6,465 & 3 & 2 & & & & & Skip & Skip & Skip & Skip & Skip & Skip \\
\hline 8,081 & 8 & 5 & & & & & & & & Skip & Skip & Skip \\
\hline 9,697 & 4 & 4 & & & & & & & Skip & Skip & Skip & Skip \\
\hline 11,313 & 0 & 0 & & & Skip & Skip & Skip & Skip & Skip & Skip & Skip & Skip \\
\hline 12,929 & 5 & 7 & & & & & & & & & & Skip \\
\hline 14,545 & 1 & 1 & & & & Skip & Skip & Skip & Skip & Skip & Skip & Skip \\
\hline 16,161 & 6 & 6 & & & & & & & & & Skip & Skip \\
\hline 17,777 & 2 & 3 & & & & & & Skip & Skip & Skip & Skip & Skip \\
\hline 19,393 & 7 & 8 & & & & & & & & & & \\
\hline 21,009 & 3 & 2 & & & & & Skip & Skip & Skip & Skip & Skip & Skip \\
\hline 22,625 & 8 & 5 & & & & & & & & Skip & Skip & Skip \\
\hline 24,241 & 4 & 4 & & & & & & & Skip & Skip & Skip & Skip \\
\hline 25,857 & 0 & 0 & & & Skip & Skip & Skip & Skip & Skip & Skip & Skip & Skip \\
\hline 27,473 & 5 & 7 & & & & & & & & & & Skip \\
\hline
\end{tabular}

Figure 2. Sample sequence of cells skipped on channel $c$ vs. quantized disturbance $q_{c}$.

\subsection{Effects of Choking on Latency}

An important property of the above approach is that, for any given link, at least one cell is always left enabled out of every $N_{\mathrm{Q}}$ subsequent ones. In fact, provided that $N_{\mathrm{S}}$ and $N_{\mathrm{Q}}$ are prime, by considering any $N_{\mathrm{Q}}$-adjacent cells allocated to the link, they are characterized by different values of $q$ (and thus $q^{\prime}$ ) drawn in the range $\left[0, N_{\mathrm{Q}}-1\right]$. Even in the most unfavorable case when $q_{k}=N_{\mathrm{Q}}-1, \forall k \in[11,26]$ (that is, choking takes place to the maximum extent on all channels), the cell, among the $N_{\mathrm{Q}}$ considered ones, for which $q^{\prime}=N_{\mathrm{Q}}-1$ is not skipped. This means that the transmission latency in ACCS cannot grow more than $N_{\mathrm{Q}}$ times with respect to TSCH, that is, it is upper bounded by

$$
d_{\max }=N_{\mathrm{Q}} \cdot\left(1+R_{\mathrm{L}}\right) \cdot N_{\mathrm{S}} \cdot T_{\mathrm{slot}}=N_{\mathrm{Q}} \cdot\left(1+R_{\mathrm{L}}\right) \cdot T_{\mathrm{sf}} .
$$

For example, if $N_{\mathrm{S}}=101, T_{\text {slot }}=20 \mathrm{~ms}, R_{\mathrm{L}}=15$, and $N_{\mathrm{Q}}=9$, then latency cannot exceed $290.88 \mathrm{~s}$. As a consequence, if packet transmissions on any link to (or from) the root are spaced by at least $5 \mathrm{~min}$, then the transmission of every new packet with ACCS starts for sure after the previous one is delivered (or dropped, if the retry limit is exceeded), causing no queuing in intermediate relay motes.

When the sampling periods of applications are large enough, so that the above assumption holds on average, the size of the queues remains typically low enough. In such conditions, buffer overrun never occurs, and ACCS is not likely to bring any negative effects on communication reliability.

\subsection{Normalization to the Best Channel}

The above mechanism considers every channel separately. While achieving the most saving for what concerns the ability to reduce the number of transmission attempts, doing so has some drawbacks. For example, let us analyze the case when all available channels are affected by a non-negligible amount of disturbance. In this case, all $q_{k}$ values selected by ACCS are strictly greater than 0 , which means that every channel is choked. However, skipping some cells on a bad channel is pointless if all the other channels are equally bad, since no improvements can be expected in this case from choking.

The above phenomenon can be tackled by considering the relative levels of disturbance among channels in the place of absolute ones. To keep this "normalization" as simple as possible (we should not forget that it must be implemented on motes with very low processing power), we decided to modify the set of values used by the shaper block for 
chocking channels, we now term $\left\{q_{k}^{\prime}\right\}$, by subtracting the minimum $q_{\text {min }}$ among them. In particular, for channel $c$,

$$
q_{c}^{\prime}=q_{c}-\min _{k \in(11, \ldots, 26)} q_{k}
$$

This means that every such value is displaced by an offset (the same for all channels of a link), which is evaluated dynamically in such a way that no cells are skipped on the best channel(s), irrespective of the related quality. For example, if the least disturbed channel suffers from a failure probability equal to $30 \%$, all other channels for which $\hat{\epsilon}_{c}$ is close to the lower bound 0.3 will not be choked in any way, and the amount of skipped cells increases progressively as disturbance grows higher.

As we will see, normalization permits to lower the ACCS latency in a consistent way, although conventional TSCH remains the fastest option, as no transmission opportunities are ever skipped, not even on very bad channels, and a residual probability to succeed is likely to exist for them. On the other hand, the ability to decrease power consumption may be slightly impaired by normalization, since the choking of very bad channels is less pronounced than when conventional ACCS (without normalization) is used, meaning that a larger fraction of attempts will fail.

\subsection{Receiver Side}

The above mechanism is conceived to operate on the transmitting side of the link only, and the benefits it brings consist in reducing the number of retries, since attempts on bad channels are often skipped. On the receiving side, idle listening occurs every time a cell remains unused because either there is no frame pending for transmission or the cell is skipped by ACCS. Since the fraction of used cells on a link only depends on the frame generation rate and the average number of retries, which, in turn, is related to the disturbance level and not on choking (that can simply defer part of the transmission opportunities), we can state that ACCS does not affect power consumption on the receiver side of the link. Idle listening is a known problem of TSCH, which can be faced by mechanisms like listening suspension performed at the MAC layer and, more in general, PRIL.

So that the maximized saving can be achieved concerning energy consumption, the receiver can be kept synchronized with the transmitter for what concerns the channelchocking mechanism. In particular, every time the transmitter computes a new $q_{c}$ estimate for a link, its value can be sent to the receiver so that it can disable listening in those cells that will be skipped. While reducing power consumption on the receiver side, this approach may suffer from non-negligible issues when, for whatever reason, coherence is lost and the values of $q_{c}$ seen by the transmitter and the receiver differ. In this case, in fact, the receiver can be mistakenly down at the time the transmitter is sending a frame, hence reducing the number of available attempts per frame and, in turn, the communication reliability. Making the above technique reliable without increasing appreciably the amount of information exchanged between the two sides of a link is an open question that will be faced in future works.

\section{Channel Estimation}

Preliminary experiments were performed aimed at evaluating the ability of moving averages to filter the outcomes of transmission attempts and to satisfactorily estimate the failure probability on every channel of a link. The two solutions described above were considered, that is, SMA and EMA. The former is parameterized by the number $M$ of past samples to be averaged, whereas the latter by weight $\alpha$. A quite generic way to describe the wireless spectrum of a link, and in particular of every single channel $c$, is to model it through a finite number of states, each one characterized by a certain quality of communication. In every state, transmission attempts are modeled as Bernoulli trials characterized by the related failure probability $\epsilon_{c}$. Transitions between states take place from time to time due 
to variations in the amount of interference and noise. This resembles a generalization of the Gilbert-Elliot model that relies on two or more states.

By resorting to simulation, the performance of SMA and EMA was evaluated for a specific $\epsilon_{\mathcal{c}}$ variation pattern. For simplicity, we modeled channel $c$ for a given link using four states, characterized by $\epsilon_{c}$ equal to $0.1,0.9,0.3$, and 0.7 , respectively. Transitions between states, in this order, took place every 100 transmission attempts on that channel. As an exception, the system stayed in the third state, corresponding to $\epsilon_{c}=0.3$, for 200 attempts. In other words, in the simulations we modeled $\epsilon_{c}$ as a specific piecewise constant function. Assuming $N_{\mathrm{C}}=16, N_{\mathrm{S}}=101, T_{\text {slot }}=20 \mathrm{~ms}$, and that a pending frame is always ready to be sent, implies that attempts on any channel $c$ (and hence, $\phi_{c}^{(i)}$ samples describing their outcomes) are spaced by about $32 \mathrm{~s}$. This means that in the above sample disturbance pattern, the time that the environment remained (mostly) stable between subsequent variations was at least $50 \mathrm{~min}$. Realistically, the spectrum can be satisfactorily described by referring to the cyclicity of human activities over the day (morning, afternoon, evening, and night), which are characterized by slower dynamics (several hours).

In Figure 3 the original $\epsilon_{c}^{(i)}$ pattern (solid line), as well as the $\hat{\epsilon}_{c}^{(i)}$ estimates computed through SMA and EMA, are plotted for a disturbance pattern including 1000 samples. For SMA we selected $M=12$, while for EMA we set $\alpha=0.12$ (they will be shown to be optimal values). The sequence $\left(\phi_{c}^{(i)}\right)$ of transmission outcomes is not shown in the figure, as it visually bears little information (it is just a random sequence of one thousand Boolean values). As can be qualitatively observed, filters are able to track satisfactorily the failure probability, with some delay. Moreover, jitters appear, whose presence can be easily observed when the failure probability is stuck at a fixed value, because of the Bernoulli process we employed to model attempts. Increasing $M$ or decreasing $\alpha$ lowers jitters, but makes filters unable to track spectrum variations promptly. An optimal value can be determined for filter parameters, which depends on the dynamics of disturbance.

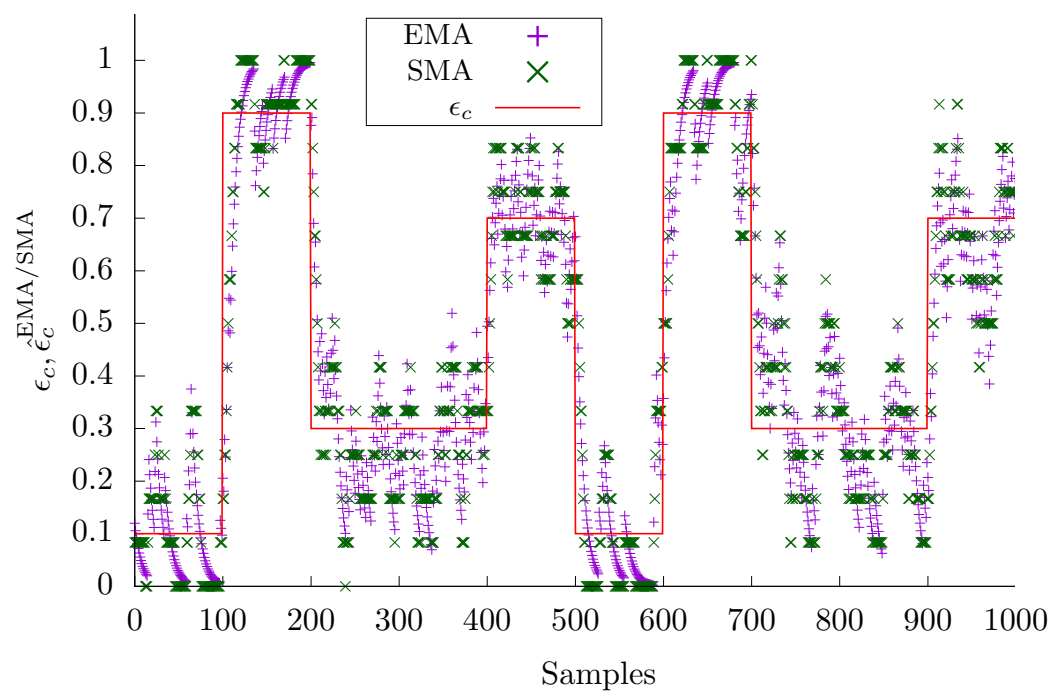

Figure 3. Real and estimated (SMA/EMA) values of $\epsilon_{c}$ vs. time (sample) $i$.

To quantitatively evaluate the effect of parameters on filtering, simulations were run by repeating our basic disturbance pattern 200 times (that is, by considering 100,000 transmission attempts overall). In every run, the root mean square error (RMSE) between $\epsilon_{c}$ and $\hat{\epsilon}_{c}$ was calculated for different values of $\alpha$ and $M$. Results are reported in Table 2. As can be seen, optimal parameter values actually exist that, for the specific sample disturbance pattern we considered, minimize RMSE. In fact, when the cutoff frequency of the low-pass filter is too high, variability of the Bernoulli process causes high jitters (and errors) on the estimated $\hat{\epsilon}_{c}$. Conversely, when it is set too low, variations in $\epsilon_{c}$ are not tracked promptly enough, making any mechanism that relies on them (such as ACCS) temporarily ineffective. 
Whatever the case, once the parameters of the filter are suitably set, a reduction in the dynamics of disturbance leads to a better estimation. Interestingly, a range of values exists for every parameter, centered around the optimum, where RMSE does not vary appreciably. We will not investigate this aspect further, since we are not interested in obtaining the best estimation for $\epsilon_{c}$ according to RMSE. In theory, what should be looked for is a filter that provides the best results for the metrics we consider for assessing ACCS communication performance. In reality, we just need a coarse analysis to find the filter parameters that offer a reasonably low RMSE. Selecting a non-optimal filter just makes ACCS behavior worse, which means that the results reported in the analysis below are pessimistic under this respect. This is not a limiting factor, as the exact dynamics of the spectrum are seldom available in advance.

Table 2. RMSE for $\epsilon_{c}$ estimations with EMA and SMA filters $\left(\hat{\epsilon}_{c}^{\mathrm{EMA}}\right.$ and $\left.\hat{\epsilon}_{c}^{\mathrm{SMA}}\right)$.

\begin{tabular}{cccc}
\hline & EMA & & \multicolumn{2}{c}{ SMA } \\
\hline$\alpha$ & RMSE & $\boldsymbol{M}$ & RMSE \\
\hline 0.050000 & 0.179107 & 4 & 0.207358 \\
0.100000 & 0.146344 & 8 & 0.163764 \\
$0.120000^{*}$ & 0.143827 & 10 & 0.157549 \\
0.150000 & 0.144726 & $12^{*}$ & 0.155372 \\
0.200000 & 0.152609 & 16 & 0.157396 \\
0.250000 & 0.164097 & 20 & 0.163598 \\
0.300000 & 0.177126 & 32 & 0.190030 \\
\hline
\end{tabular}

*optimum value for the considered configuration.

\section{Communication Performance}

An experimental campaign was carried out aimed to evaluate the effects of choking on communication performance, to understand if and under what conditions ACCS may bring any real benefits. A very simple program written in the $C$ language was exploited to this extent, which simulates the behavior of a link with a single dedicated cell operated according to either the conventional TSCH mechanism, the conventional choking mechanisms, or the normalized chocking mechanism.

\subsection{Metrics}

Comparison relied on several metrics related to power consumption, responsiveness, and reliability. Concerning power consumption, we counted the number $a_{j}$ of attempts performed for every transmitted frame $j$. Both successfully delivered frames and those that were lost because the retry limit was exceeded were considered, since energy is consumed in either case. To better highlight the behavior of ACCS, the retry limit in our experiments was set to $R_{\mathrm{L}}=7$ (that is, at most, 8 transmission attempts can be performed for any given frame before it is discarded). This value sits in between the values customarily set in TSCH for this parameter, that is, 3 (in the early literature) and 15 (in the current implementation). By using a suitable power consumption model, such as the ones described in $[9,24]$, the overall energy spent for every frame transmission (all attempts included) can be easily evaluated. Below, we preferred to stick on the average number of transmission attempts per frame, and not on energy, because this value does not depend on the specific implementation of the mote and, in particular, of its radio transceiver. At any rate, these two values are related by a linear law. In particular, we calculated the mean value and standard deviation of the sequence $\left(a_{j}\right)$ of experimental samples, denoted $\mu_{a}$ and $\sigma_{a}$, respectively. 
The second metric we took into account is the transmission latency, denoted $d_{j}$, which is defined only for those frames that were successfully delivered to destination. A relevant contribution to the latency is the number of retries made to deliver the frame, which equals $a_{j}-1$. In fact, on every retry the latency increases by one slotframe $T_{\mathrm{sf}}$. Actually, when choking is active, also the skipped cells must be taken into account, whose number for frame $j$ is denoted $s_{j}$. Every frame transmission is additionally affected by a waiting time, due to the fact that slotframe repetition and actual sampling are typically not synchronized. Its contribution to the overall delay is not fixed, but is always upper bounded by the slotframe duration and, on average, it amounts to $T_{\mathrm{sf}} / 2$ [14]. We neglected delays due the communication stack in both the receiver and the transmitter, as well as propagation delays for signals over the air, because they do not depend on the communication protocol.

Since we are interested in the worst case, we considered a proper upper bound on latency by assuming that the maximum waiting time is always experienced. It can be expressed by rounding the number of slotframes, which elapse between the frame transmission request and the slot when the corresponding ACK frame is received from the destination to the next whole number. This upper bound can be expressed as $\hat{\delta}_{j} T_{\text {sf }}$, where $\hat{\delta}_{j}=a_{j}+s_{j}$. For example, in the case that the frame is delivered at the first attempt and no cells are skipped, $\hat{\delta}_{j}$ is equal to 1 (one slotframe). Statistics concerning $\hat{\delta}_{j}$ were evaluated for every experiment, including the mean value $\mu_{\hat{\delta}}$, the standard deviation $\sigma_{\hat{\delta}}$ (which provides an indication about jitters), and the maximum value $\hat{\delta}_{\text {max }}$. Interestingly, the actual mean value for latency can be determined as $\mu_{d} \simeq \mu_{\hat{\delta}} T_{\text {sf }}-T_{\text {sf }} / 2$, while for the worst-case latency $d_{\text {max }} \simeq \hat{\delta}_{\text {max }} T_{\text {sf }}$.

The latter metric is reliability, expressed as the fraction $P_{\mathrm{L}}$ of frames that went lost because no acknowledgments were received while performing the allowed attempts. When ACK frames are repeatedly corrupted, chances are that a correctly received frame is considered failed by the transmitter. From a practical viewpoint this is not a problem, as neglecting this event simply makes our analysis slightly pessimistic.

\subsection{Spectrum Model}

For what concerns disturbance affecting communication, we assumed that transmission attempts are independent and can be modeled as Bernoulli trials. In particular, every channel $c$ is characterized by a failure probability $\epsilon_{c}$. Thanks to the mixed time-frequency diversity in TSCH, this is usually a more than reasonable approximation (see [14]). As in the current OpenWSN implementation on OpenMotes B, we assumed that 16 channels are exploited by TSCH in the $2.4 \mathrm{GHz}$ band, labeled from 11 to 26 according to [5].

To simplify the definition of experiments, we considered groups of four adjacent WSN channels (e.g., 11, 12, 13, and 14), and we set the failure probability for all the channels in every such group at the same value (for the example, $\epsilon_{11-14}$ ). In other words, the spectrum seen by TSCH is completely characterized by the tuple $\left\langle\epsilon_{11-14}, \epsilon_{15-18}, \epsilon_{19-22}, \epsilon_{23-26}\right\rangle$. Such an assumption does not imply any particular limitations on our analysis and is perfectly coherent with the interference generated by co-located $20 \mathrm{MHz}$ channels in well-configured Wi-Fi networks tuned on channels 1, 5, 9, and 13.

In the following, we chose for ACCS a low-pass filter based on EMA, where $\alpha=0.05$. Doing so provides an acceptable trade-off between responsiveness in tracking changes of the spectrum conditions and the ability to provide smooth results.

The values of the main parameters used in the experiments, concerning both the network protocol and the wireless environment are reported in Table 3. 
Table 3. Parameters used in the experiments (protocol and environmental).

\begin{tabular}{lcr}
\hline Parameter & Symbol & Value \\
\hline Slotframe size (in slots) & $N_{\mathrm{S}}$ & 11 \\
Number of channels & $N_{\mathrm{C}}$ & 16 \\
Number of quantization levels & $N_{\mathrm{Q}}$ & 9 \\
Retry limit & $R_{\mathrm{L}}$ & 7 \\
Weight for EMA low-pass filter & $\alpha$ & 0.05 \\
Failure probability (Wi-Fi interf. ch. 1) & $\epsilon_{11-14}$ & $0.1^{*} / 0.9^{* *} / 0.1^{\dagger}$ \\
Failure probability (Wi-Fi interf. ch. 5) & $\epsilon_{15-18}$ & $0.3^{*} / 0.3^{* *} / 0.1^{\dagger}$ \\
Failure probability (Wi-Fi interf. ch. 9) & $\epsilon_{19-22}$ & $0.7^{*} / 0.7^{* *} / 0.1^{+}$ \\
Failure probability (Wi-Fi interf. ch. 13) & $\epsilon_{23-26}$ & $0.1^{*} / 0.9^{* *} / 0.1^{\dagger}$ \\
\hline${ }^{*}$ mild;** heavy; + negligible disturbance conditions (steady-state analysis).
\end{tabular}

\subsection{Steady-State Analysis}

Experiments were first performed in steady-state conditions, where the failure probability on every channel was set to a fixed value for the whole duration of the test. Three conditions were considered: the first mimics a mildly disturbed spectrum, described as $\epsilon_{11-14}=0.1, \epsilon_{15-18}=0.3, \epsilon_{19-22}=0.7$, and $\epsilon_{23-26}=0.1$. Instead, the second refers to a heavily disturbed spectrum, where failure probabilities were $\epsilon_{11-14}=0.9, \epsilon_{15-18}=0.3$, $\epsilon_{19-22}=0.7$, and $\epsilon_{23-26}=0.9$. These are clearly quite high values, but it must be remembered that the ACCS mechanism we are proposing is aimed explicitly at coping with such conditions. Finally, the third condition models the case when disturbance is negligible. In this case, $\epsilon_{11-14}=\epsilon_{15-18}=\epsilon_{19-22}=\epsilon_{23-26}=0.1$.

For each spectrum condition, three experiments were performed, considering conventional TSCH, ACCS, and normalized ACCS. Every experiment lasted for ten million cells. For a slot time equal to $20 \mathrm{~ms}$, this means more than two days in the real world. We assumed that frames are sent by the application back to back. While this is not the way motes typically operate (in systems with slow dynamics, samplings are usually spaced by tens of minutes, and even hours), the results are not affected because of our hypothesis that attempts are statistically independent. The duration of the slotframe was set equal to 11 slots. Doing so increases the number of transmitted frames and improves the reliability of statistics. Results are reported in Table 4, where the upper, middle, and lower parts of the table refer to mild, heavy, and negligible interference conditions, respectively.

Table 4. Steady-state analysis (mild, heavy and negligible disturbance).

\begin{tabular}{|c|c|c|c|c|c|c|c|}
\hline \multirow[t]{3}{*}{ Technique } & \multirow[t]{3}{*}{ Disturb. } & \multicolumn{2}{|c|}{ Tries (\#) } & \multicolumn{3}{|c|}{ Latency (Slotframes) } & \multirow{3}{*}{$\begin{array}{c}\text { Losses } \\
\% \\
P_{\mathrm{L}}\end{array}$} \\
\hline & & (avg) & (std) & (avg) & (std) & (worst) & \\
\hline & & $\mu_{a}$ & $\sigma_{a}$ & $\mu_{\hat{\delta}}$ & $\sigma_{\hat{\delta}}$ & $\hat{\delta}_{\max }$ & \\
\hline Default TSCH & & 1.42859 & 0.50597 & 1.42853 & 0.50556 & 8 & 0.0009 \\
\hline Choked norm. & mild & 1.27902 & 0.32618 & 1.70465 & 0.88144 & 11 & 0.0002 \\
\hline Choked & & 1.27901 & 0.32616 & 1.70484 & 0.88171 & 11 & 0.0002 \\
\hline Default TSCH & & 3.18516 & 4.46910 & 2.96537 & 3.56654 & 8 & 4.3656 \\
\hline Choked norm. & heavy & 2.38124 & 2.51568 & 4.47979 & 10.02960 & 22 & 0.8030 \\
\hline Choked & & 2.08231 & 1.84247 & 6.00560 & 19.19481 & 32 & 0.3266 \\
\hline Default TSCH & & 1.11131 & 0.12393 & 1.11131 & 0.12393 & 6 & 0.0 \\
\hline Choked norm. & neglig. & 1.11139 & 0.12424 & 1.16392 & 0.18600 & 7 & 0.0 \\
\hline Choked & & 1.11139 & 0.12424 & 1.16392 & 0.18600 & 7 & 0.0 \\
\hline
\end{tabular}

As can be seen in the middle part, where disturbance is objectively heavy, conventional $\mathrm{TSCH}$ features the shortest latency. However, more than three attempts per frame are performed on average in these conditions, which implies that the power consumption on the transmitter side is three times higher than when there is no disturbance at all (so that 
transmissions always succeed at the initial attempt). Reliability is only partially satisfactory, as $4.37 \%$ of the frames are lost.

When choking is considered, the number of attempts per frame decreases noticeably (about two are needed, on average), and the same holds for power consumption (which means a $35 \%$ reduction in the energy spent by the radio module of the transmitter). On the downside, latency increases sensibly, and is on average twice as much as TSCH. Even worse are jitters, as can be seen by observing the standard deviation of the latency, as well as its maximum value. As we said above, this is not a problem for the use cases we are considering. Unlike control loops with tight timing constraints (which, indeed, rely seldom on WSNs), latency is mostly irrelevant for applications that perform sensing, especially when timestamps associated to samples are taken on motes. The reliability of ACCS is much better than TSCH. In fact, by skipping bad channels, the fraction of dropped frames decreased by more than one order of magnitude.

When normalization was enabled for ACCS, the results we obtained sat in between the two above cases. In particular, power consumption increased by about $15 \%$ over basic choking ( $25 \%$ less than TSCH), but the mean and maximum latency, as well as the related standard deviation, were noticeably lower. Reliability was, again, noticeably better than $\mathrm{TSCH}$, although not as much as in the previous case.

The very same behavior is observed in the upper part of the table, where the link suffered from mild disturbance on half of the channels. In this case, the differences between conventional and choked TSCH for what concerns power consumption and latency are less pronounced. As can be seen, normalization hardly has any noticeable effects on choking. This is because of the channels with the best quality (i.e., characterized by the lower disturbance). In fact, the failure probability estimated for them almost always led to a value for $q_{\min }$ equal to (or near to) 0 , which means that most of the times $q_{c}^{\prime}$ values were non-actually displaced by normalization. Interestingly, the ability to skip bad channels managed to improve reliability noticeably. In fact, the fraction of lost frames when choking is enabled decreased more than four times compared to TSCH.

Finally, in the lower part of the table, it can be observed that when disturbance is quite low, the effect of ACCS is barely noticeable: almost the same results as TSCH were obtained for power consumption, with a modest increase in the latency. This is because, with such a low level of disturbance, choking is performed infrequently. Having the same amount of disturbance on all channels did not help, as it makes selective choking useless. For the same reasons, normalization had no visible effect. These are desirable results, as in typical, benign conditions, ACCS basically retains the behavior of TSCH.

The results in Table 4 are presented as bar charts in Figure 4 to ease comparison among the different approaches. In particular, every plot refers to a specific quantity: the number of attempts (average and standard deviation), upper bound on latency (average, standard deviation, and worst case), and packet-loss ratio. 

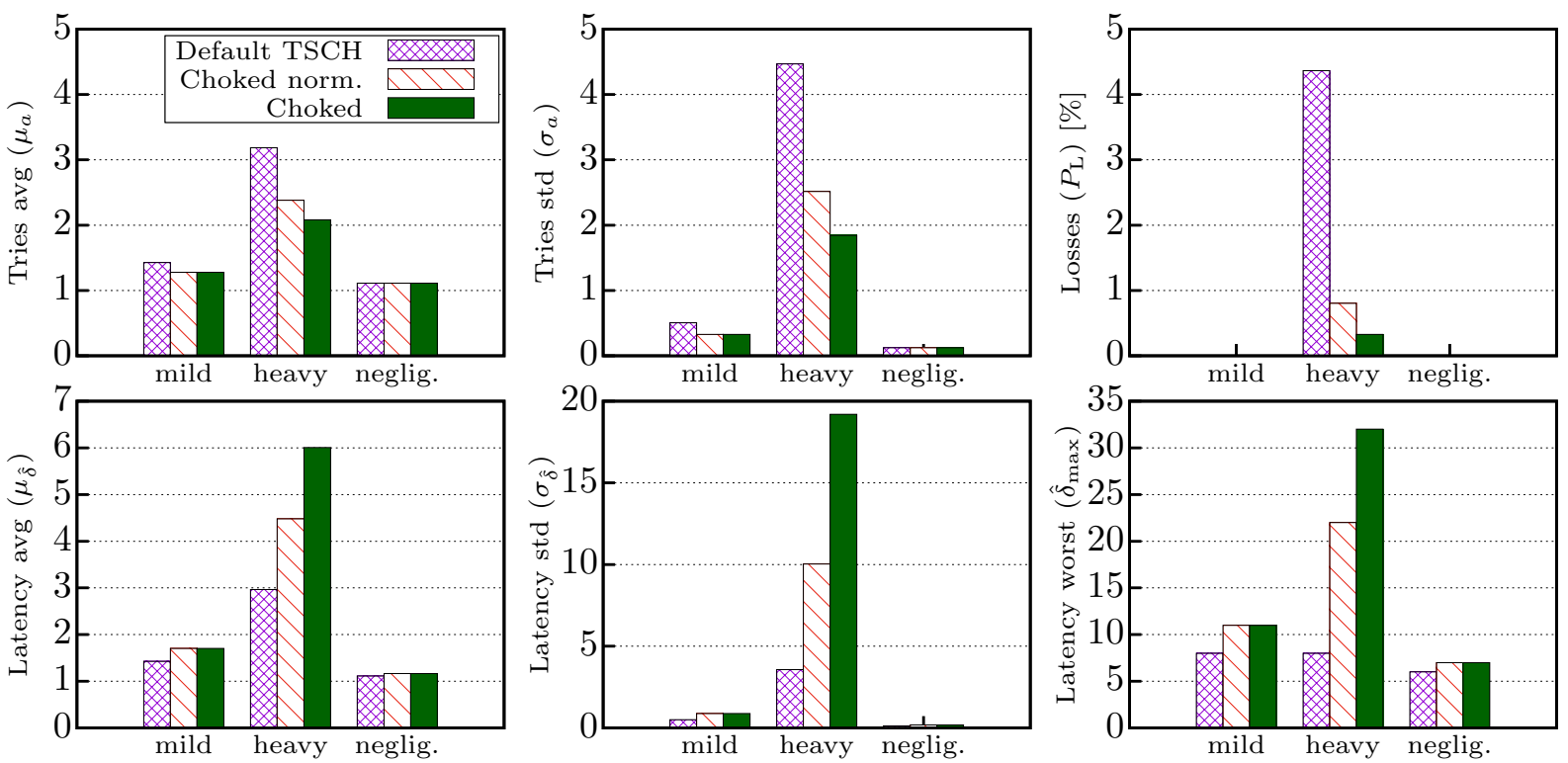

Figure 4. Performance indicators about communication for the different approaches (TSCH, ACCS, and normalized ACCS) in the three considered operating conditions (mild, heavy, and negligible disturbance).

\subsection{Transient Analysis}

This experiment was aimed at determining whether or not making choking work on the estimated probability may bring tangible benefits when a dynamically changing environment is taken into account. As in the case above, the experiments lasted for ten million cells, and the failure probabilities for (groups of) channels were changed four times in this interval. Initially, $\epsilon_{11-14}=0.1, \epsilon_{15-18}=0.3, \epsilon_{19-22}=0.7$, and $\epsilon_{23-26}=0.1$. Then, the failure probabilities for all channels were progressively raised up to 0.9 , with the exception of $\epsilon_{19-22}$, that remained at 0.7 . This happened at cell 2,500,000 for $\epsilon_{23-26}$, at cell $5,000,000$ for $\epsilon_{11-14}$, and at cell 7,500,000 for $\epsilon_{15-18}$, leading to four sub intervals.

The metrics defined above for steady-state analysis were evaluated again to compare $\mathrm{TSCH}$ and the two choking mechanisms (with and without normalization). In the latter cases, the choking mechanism was driven either by the real failure probability $\epsilon$ (the same we used to generate the outcomes of attempts, modeled as Bernoulli trials) or by the probability estimated by applying a low-pass filter on past outcomes. In particular, we opted again for the use of EMA by setting $\alpha=0.05$. Results obtained when ACCS was driven directly by $\epsilon_{\mathcal{c}}$ values (and not by $\hat{\epsilon}_{c}$ estimates) can be seen as benchmarks. In fact, doing so permitted to prevent the effects due to estimation errors (both jitters and slowness in tracking the failure probability in dynamical varying environments), which negatively affect ACCS behavior.

Results are reported in Table 5. As can be seen, even in the case when the disturbance level varied with time, ACCS managed to reduce the average number of retries (and hence, power consumption) at the expense of an increased latency. Moreover, it permitted, again, to reduce the number of lost frames, thus leading to better communication reliability. Additionally the effects of normalization on ACCS resemble the steady-state case. 
Table 5. Transient analysis (disturbance grows as the time passes).

\begin{tabular}{lccccccc}
\hline Technique & Disturb. & \multicolumn{2}{c}{ Tries (\#) } & \multicolumn{2}{c}{ Latency (Slotframes) } & Losses \\
\hline & & (avg) & (std) & (avg) & (std) & (worst) & $\%$ \\
& & $\mu_{\boldsymbol{a}}$ & $\sigma_{\boldsymbol{a}}$ & $\boldsymbol{\mu}_{\hat{\delta}}$ & $\sigma_{\hat{\delta}}$ & $\hat{\delta}_{\text {max }}$ & $\boldsymbol{P}_{\mathbf{L}}$ \\
\hline Default TSCH & - & 2.326765 & 3.577665 & 2.090291 & 2.329300 & 8 & 4.0014 \\
Choked norm. & estimat. & 1.929775 & 2.804046 & 2.721700 & 5.020538 & 22 & 3.1911 \\
Choked norm. & real * & 1.866562 & 2.597595 & 2.665680 & 5.084163 & 36 & 2.8788 \\
Choked & estimat. & 1.565869 & 1.227635 & 3.140490 & 18.018015 & 67 & 0.7421 \\
Choked & real $^{*}$ & 1.518178 & 1.068892 & 3.061340 & 19.575816 & 62 & 0.5735 \\
\hline
\end{tabular}

${ }^{*}$ experiments based on the real $\epsilon$ value are only included as a term of reference.

Concerning comparison with the benchmarks based on true $\epsilon_{\mathcal{c}}$ values, we can observe that for the analyzed case, characterized by a slowly varying spectrum, working on $\hat{\epsilon}_{c}$ estimates did not lower ACCS performance appreciably. Overall, we managed to increase the probability that frames are successfully delivered to destination and to save energy at the same time, which can be useful in several contexts where applications operate slowly (several tens of minutes between data exchanges); the main goal is extending the lifetime of motes.

The plots in Figures 5 and 6 show the number of transmission attempts (that is related to power consumption) and the upper bound on latency (in slotframes), respectively, for conventional TSCH, ACCS, and normalized ACCS. A mobile average over 100 samples was applied to make plots smoother. Again, it can be seen that when disturbance increases, selectively skipping bad cells permits to lower, on average, the number of attempts.

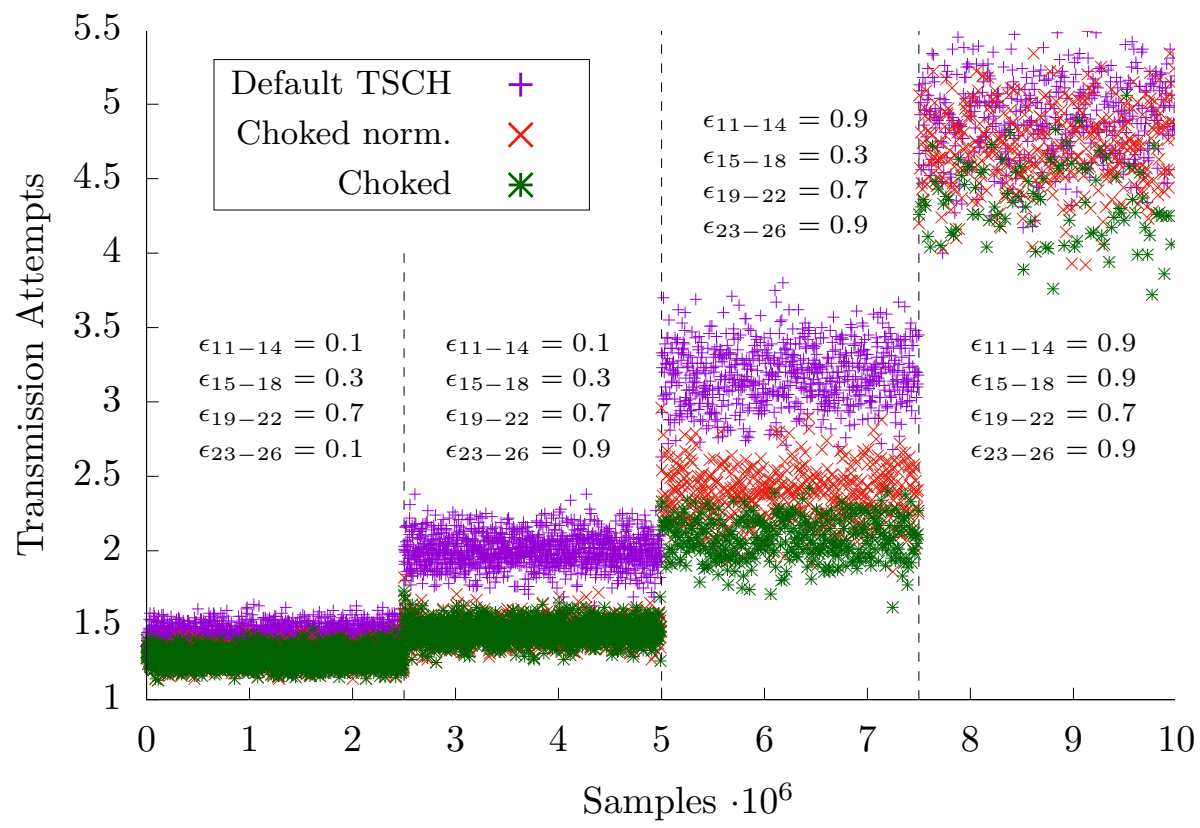

Figure 5. Number of transmission attempts per frame vs. time (sample). 


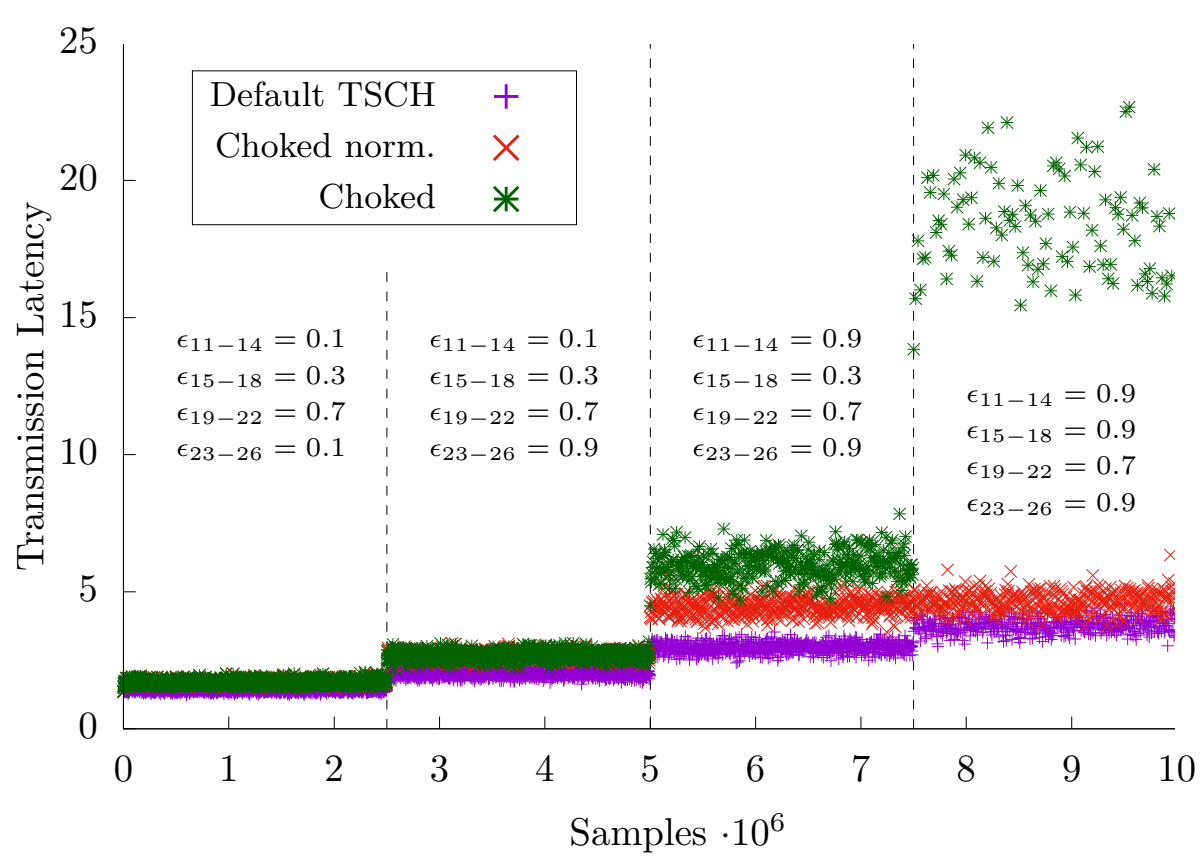

Figure 6. Frame transmission latency vs. time (sample).

\section{Conclusions}

Thanks to its MAC mechanism based on time slotting, TSCH achieves low duty cycles for the radio module of motes and, hence, reduced power consumption. At the same time, channel hopping lowers the probability that frames are lost consistently, therefore ensuring higher reliability. In this paper, a mechanism is described and evaluated that makes TSCH ultra-low power, and, hence, able to suit the needs of unattended distributed systems deployed without a fixed network infrastructure, including those that rely on the Internet of Things paradigm for, e.g., environmental monitoring, diagnostics of industrial plants, and smart agriculture, where a number of nodes are powered on batteries whose lifetime must last as long as possible.

This mechanism, which we termed ACCS, operates on a per-link basis and provides sort of a probabilistic blacklisting, where better quality channels are more likely to be used for transmission on links. First, ACCS evaluates the quality of all the channels of every link. Such quality can be profitably described by means of the failure probability computed from the fraction of attempts for which an ACK is received. Then, the capacity of every channel of any given link is separately choked, so that the lower its failure probability, the most it will be actually exploited for transmission attempts. From a practical point of view, cells allocated to the link are selectively skipped by means of a pseudo-probabilistic scheme, in such a way as to favor channels suffering from a low disturbance. Badly disturbed channels are nevertheless used at a reduced rate so that their quality can be still assessed.

The ACCS performance, in terms of reliability, power consumption, and latency, was evaluated by means of a very simple network simulator and compared to conventional TSCH. Results are quite encouraging, and show that both power consumption and reliability can be improved tangibly. The only downside is that transmission latency may increase sensibly, since part of the transmission opportunities are purposely skipped. However, when applications with slow timing requirements are taken into account, such as those devoted to collect information from a physical system (i.e., when control loops are not closed over the network), this is not a severe limitation. For example, when parameters are selected as in our experiments, ACCS never makes the latency grow more than nine times with respect to TSCH. Last but not least, the proposed mechanism is extremely simple, which has two advantages: first, a streamlined implementation is possible, which fits the scarce processing resources of motes, and second, good robustness is also expected, which 
means that the network behavior is unlikely to worsen because of rare and unexpected events.

Our future work will include a more thorough analysis of the effects of both the filtering technique and the choking strategy on communication performance.

Author Contributions: All authors, G.C., S.S., and A.V., contributed equally to the paper. All authors have read and agreed to the published version of the manuscript.

Funding: This research received no external funding.

Conflicts of Interest: The authors declare no conflict of interest.

\author{
Abbreviations \\ The following abbreviations are used in this manuscript: \\ TSCH Time Slotted Channel Hopping \\ ACCS Adaptive Channel Capacity Shaping
}

\title{
References
}

1. Flexible Self-Charging Battery under Development. Available online: https://www.advancedbatteriesresearch.com/articles/22 081/flexible-self-charging-battery-under-development (accessed on 11 January 2022).

2. Sethi, P.; Sarangi, S.R. Internet of Things: Architectures, Protocols, and Applications. J. Electr. Comput. Eng. 2017, 2017, 9324035. doi: $10.1155 / 2017 / 9324035$ [CrossRef]

3. Chen, Q.; Gursoy, M.C. Energy-efficient modulation design for reliable communication in wireless networks. In Proceedings of the 2009 43rd Annual Conference on Information Sciences and Systems, Baltimore, MD, USA, 18-20 March 2009; pp. 811-816. [CrossRef]

4. Wen, M.; Cheng, X.; Yang, L. Optimizing the energy efficiency of OFDM with index modulation. In Proceedings of the 2014 IEEE International Conference on Communication Systems, Macau, China, 19-21 November 2014; pp. 31-35. [CrossRef]

5. IEEE Std 802.15.4-2020 (Revision of IEEE Std 802.15.4-2015); IEEE Standard for Low-Rate Wireless Networks. IEEE: New York, NY, USA, 23 July 2020; pp. 1-800. [CrossRef]

6. Vilajosana, X.; Watteyne, T.; Chang, T.; Vučinić, M.; Duquennoy, S.; Thubert, P. IETF 6TiSCH: A Tutorial. IEEE Commun. Surv. Tutorials 2020, 22, 595-615. [CrossRef]

7. Scanzio, S.; Vakili, M.G.; Cena, G.; Demartini, C.G.; Montrucchio, B.; Valenzano, A.; Zunino, C. Wireless Sensor Networks and TSCH: A Compromise Between Reliability, Power Consumption, and Latency. IEEE Access 2020, 8, 167042-167058. [CrossRef]

8. Juc, I.; Alphand, O.; Guizzetti, R.; Favre, M.; Duda, A. Energy consumption and performance of IEEE 802.15.4e TSCH and DSME. In Proceedings of the 2016 IEEE Wireless Communications and Networking Conference, Doha, Qatar, 3-6 April 2016; pp. 1-7. [CrossRef]

9. Cena, G.; Scanzio, S.; Valenzano, A. Enabling Listening Suspension in the Time Slotted Channel Hopping Protocol. In Proceedings of the 2021 17th IEEE International Conference on Factory Communication Systems (WFCS), Linz, Austria, 9-11 June 2021; pp. 19-26. [CrossRef]

10. Scanzio, S.; Cena, G.; Valenzano, A.; Zunino, C. Energy Saving in TSCH Networks by Means of Proactive Reduction of Idle Listening. In Ad-Hoc, Mobile, and Wireless Networks; Grieco, L.A., Boggia, G., Piro, G., Jararweh, Y., Campolo, C., Eds.; Springer International Publishing: Cham, Switzerland, 2020; pp. 131-144.

11. Scanzio, S.; Cena, G.; Seno, L.; Valenzano, A. Robustness and Optimization of PRIL Techniques for Energy Saving in TSCH Networks. In Proceedings of the 26th IEEE International Conference on Emerging Technologies and Factory Automation (ETFA 2021), Vasteras, Sweden, 7-10 September 2021; pp. 1-8. [CrossRef]

12. Selvi, M.; Santhosh Kumar, S.V.N.; Ganapathy, S.; Ayyanar, A.; Khanna Nehemiah, H.; Kannan, A. An Energy Efficient Clustered Gravitational and Fuzzy Based Routing Algorithm in WSNs. Wirel. Pers. Commun. 2021, 116, 61-90. [CrossRef]

13. Elwekeil, M.; Abdalzaher, M.S.; Seddik, K. Prolonging smart grid network lifetime through optimising number of sensor nodes and packet length. IET Commun. 2019, 13, 2478-2484.

14. Cena, G.; Demartini, C.G.; Ghazi Vakili, M.; Scanzio, S.; Valenzano, A.; Zunino, C. Evaluating and Modeling IEEE 802.15.4 TSCH Resilience against Wi-Fi Interference in New-Generation Highly-Dependable Wireless Sensor Networks. Ad Hoc Netw. 2020, 106, 102199. [CrossRef]

15. Kotsiou, V.; Papadopoulos, G.Z.; Chatzimisios, P.; Theoleyre, F. LABeL: Link-based Adaptive BLacklisting Technique for 6TiSCH Wireless Industrial Networks. In Proceedings of the 20th ACM Int. Conference on Modelling, Analysis and Simulation of Wireless and Mobile Systems, Miami, FL, USA, 21-25 November 2017; ACM: New York, NY, USA, 2017; pp. 25-33. [CrossRef]

16. Lazarescu, M.T. Design of a WSN Platform for Long-Term Environmental Monitoring for IoT Applications. IEEE J. Emerg. Sel. Top. Circuits Syst. 2013, 3, 45-54. [CrossRef] 
17. Khoufi, I.; Minet, P.; Rmili, B. Beacon Advertising in an IEEE 802.15.4e TSCH Network for Space Launch Vehicles. In Proceedings of the 7th European Conference for Aeronautics and Aerospace Sciences (EUCASS 2017), Milan, Italy, 3 July 2017.

18. Zoppi, S.; Gürsu, H.M.; Vilgelm, M.; Kellerer, W. Reliable hopping sequence design for highly interfered wireless sensor networks. In Proceedings of the 2017 IEEE International Symposium on Local and Metropolitan Area Networks (LANMAN), Osaka, Japan, 12-14 June 2017; pp. 1-7. [CrossRef]

19. Du, P.; Roussos, G. Adaptive time slotted channel hopping for wireless sensor networks. In Proceedings of the 20124 th Computer Science and Electronic Engineering Conference (CEEC), Colchester, UK, 12-13 Sepember 2012; pp. 29-34. [CrossRef]

20. Kotsiou, V.; Papadopoulos, G.Z.; Chatzimisios, P.; Tholeyre, F. Is local blacklisting relevant in slow channel hopping low-power wireless networks? In Proceedings of the 2017 IEEE International Conference on Communications (ICC), Paris, France, 21-25 May 2017; pp. 1-6. [CrossRef]

21. Queiroz, D.V.; Gomes, R.D.; Fonseca, I.E.; Alencar, M.S.; Benavente-Peces, C. Channel Assignment in TSCH-based Wireless Sensor Networks Using Fuzzy Logic. J. Ambient. Intell. Humaniz. Comput. 2021. [CrossRef]

22. Gomes, P.H.; Watteyne, T.; Krishnamachari, B. MABO-TSCH: Multihop and blacklist-based optimized time synchronized channel hopping. Trans. Emerg. Telecommun. Technol. 2018, 29, e3223. [CrossRef]

23. Paxson, V.; Allman, M.; Chu, J.; Sargent, M. Computing TCP's Retransmission Timer. RFC 2011, 6298, 1-11. [CrossRef].

24. Vilajosana, X.; Wang, Q.; Chraim, F.; Watteyne, T.; Chang, T.; Pister, K.S.J. A Realistic Energy Consumption Model for TSCH Networks. IEEE Sens. J. 2014, 14, 482-489. [CrossRef] 